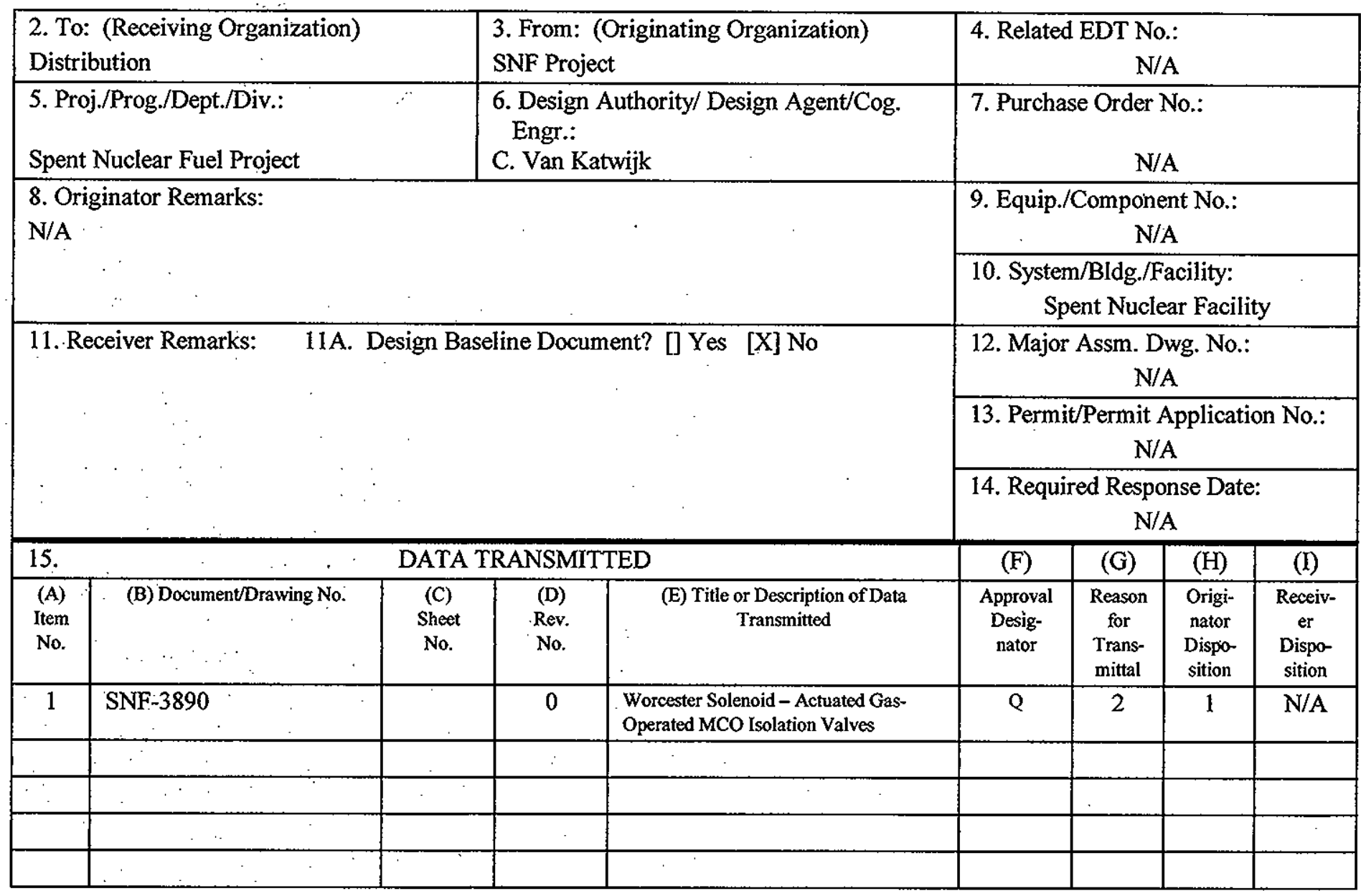

16.

KEY

\begin{tabular}{|l|ll|ll|}
\hline \multicolumn{1}{|c|}{ Approval Designator (F) } & \multicolumn{2}{|c|}{ Reason for Transmittal (G) } & \multicolumn{2}{c|}{ Disposition (H) \& (I) } \\
\hline E, S, Q, D or N/A & 1. Approval & 4. Review & 1. Approved & 4. Reviewed no/comment \\
(see WHC-CM-3-5, & 2. Release & 5. Post-Review & 2. Approved w/comment & 5. Reviewed w/comment \\
Sec.12.7) & 3. Information & 6. Dist. (Receipt Acknow. Required) & 3. Disapproved w/comment & 6. Receipt acknowledged \\
\hline
\end{tabular}

17. SIGNATURE/DISTRIBUTION

(See Approval Designator for required signatures)

\begin{tabular}{|c|c|c|c|c|c|c|}
\hline $\begin{array}{l}\text { (G) } \\
\text { Rea- } \\
\text { son }\end{array}$ & $\begin{array}{l}\text { (H) } \\
\text { Disp. }\end{array}$ & (J) Name $\quad$ (K) Signature (L) Date (M) MSIN & $\begin{array}{l}(G) \\
\text { Rea- } \\
\text { Son }\end{array}$ & $\begin{array}{l}\text { (H) } \\
\text { Disp. }\end{array}$ & (J) Name & (K) Signature (L) Date (M) MSIN \\
\hline 2 & 1 & Designated Engineer C. Van & & & & \\
\hline 2 & 1 & Design Authority J. J. Irwin & & & & \\
\hline 2 & 1 & QA T.D. Hays & & & & \\
\hline & & & & & & \\
\hline & & & & & & \\
\hline & & & & & & \\
\hline & & & & & & \\
\hline
\end{tabular}

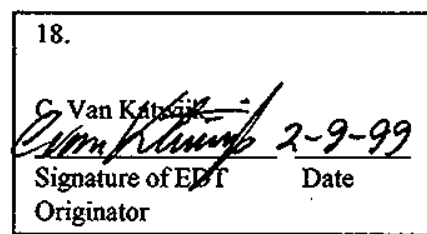

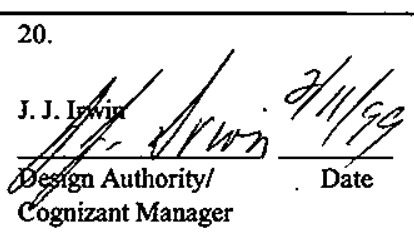

21. DOE APPROVAL (if required) Ctril. No.

D Approved

I Approved w/comments

D Disapproved w/comments
Authorized Representative Date for Receiving Organization 


\section{Worcester Solenoid - Actuated Gas-Operated MCO Isolation Valves}

Carl Van Katwijk

Numatec Hanford Co, Richland, WA 99352

U.S. Department of Energy Contract DE-AC06-96RL13200

EDT/ECN: 626263

UC: 620

Org Code: $2 \mathrm{G} 300$

B\&R Code: $39 \mathrm{EW} 40400$

Charge Code: 105559/A000

Total Pages: 14

Key Words: Isolation Valves - MCO

Abstract: Worcester Solenoid - Actuated Gas-Operated MCO Isolation Valves

CGI-SNF-D-46-1-P4-015

TRADEMARK DISCLAIMER. Reference herein to any specific commercial product, process, or service by trade name, trademark, manufacturer, or otherwise, does not necessarily constitute or imply its endorsement, recommendation, or favoring by the United States Government or any agency thereof or its contractors or subcontractors.

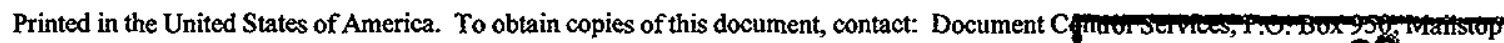
H6-08, Richland WA 99352, Phone (509) 372-2420; Fax (509) 376-4989.

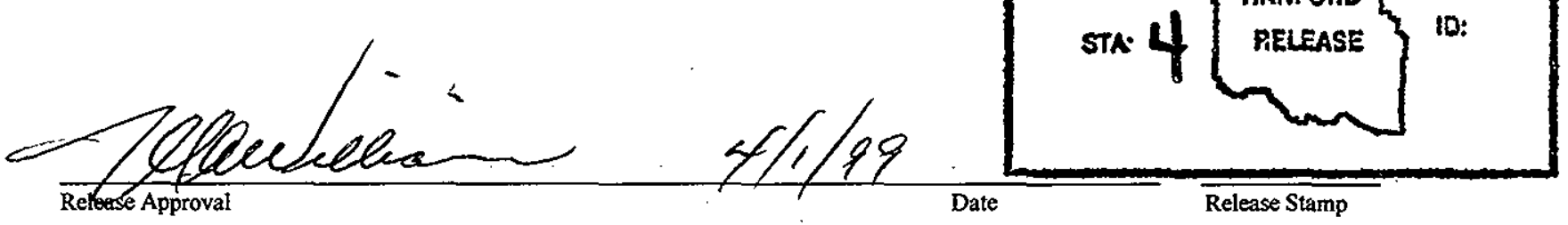

\section{Approved for Public Release}




\begin{tabular}{|l|l|}
\hline ECN No. $\frac{\text { NA }}{\text { CGINo. CGI-SNE-D-46-1-P4-015 }}$ & Page 1 of 13 \\
Title: & WORCESTER SOLENOM-ACTUATED GAS-OPERATED MCO \\
ISOLATTON VALVES & SNF 3890 \\
\hline
\end{tabular}

\begin{tabular}{|c|c|c|c|}
\hline Item No.: NA & \multicolumn{2}{|l|}{ Manufacturer: } & Supplier: \\
\hline \multicolumn{2}{|l|}{ Mfg. Part/Model No.: } & \multicolumn{2}{|l|}{ Supplier's PIN: } \\
\hline \multicolumn{4}{|l|}{ Part Description: } \\
\hline \multicolumn{4}{|l|}{ End Use Description: } \\
\hline \multicolumn{4}{|c|}{ 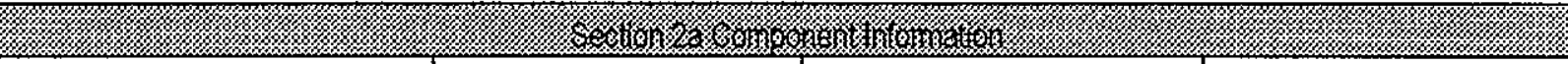 } \\
\hline $\begin{array}{l}\text { Equipment No.: GOV/SOV- } \\
1 * 03 \& 1 * 30\end{array}$ & $\begin{array}{l}\text { Specification No.: } \\
\text { W-441-P4, Rev } 2\end{array}$ & $\begin{array}{l}\text { Manufacturer: } \\
\text { Worcester Controls }\end{array}$ & Past P.O. No.: NA \\
\hline $\begin{array}{l}\text { Manufacturer's Part/ } \\
\text { Model No.: } \\
\text { 1"5966RTBW4 with } \\
\text { 151939SWM2120PBC }\end{array}$ & $\begin{array}{l}\text { Equipment Supplier (if c } \\
\text { TBD } \\
\text { : }\end{array}$ & ent from manufacturer): & $\begin{array}{l}\text { Equip. Supplier's Part No: } \\
\text { NA }\end{array}$ \\
\hline \multicolumn{4}{|c|}{$\begin{array}{l}\text { Component Description: These valves are } 1 \text { " gas-operated full-port ball valves incorporating a solenoid } \\
\text { and limit switches as integral parts of the actuator that are used in process streams within the } \\
\text { CVDF hood. The valves fail closed (on loss of pressure or electrical) to prevent MCO vent drain to } \\
\text { either reduce air inleakage or loss of He. The valves have coupling for transverse actuator } \\
\text { mounting. }\end{array}$} \\
\hline \multicolumn{4}{|c|}{ 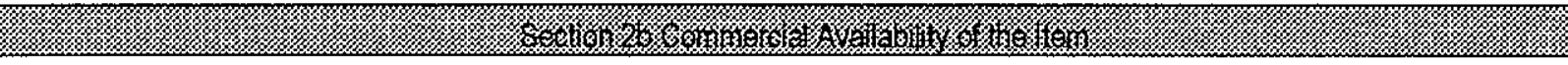 } \\
\hline \multicolumn{4}{|c|}{$\begin{array}{l}\text { 1. Is the Item available from a catalogue from a qualified NQA1 supplier } \\
\text { interface Engineer or BTR)? } \\
\text { [ ] YES (go to } \# 2 \text { below) } \\
\text { [X] NO (go to procedure step 5.3.2, proceed to dedicate ltem) }\end{array}$} \\
\hline \multicolumn{4}{|c|}{ 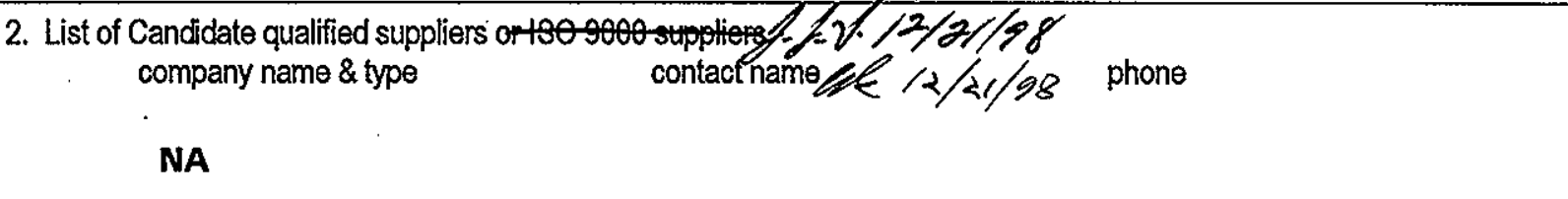 } \\
\hline \multicolumn{4}{|c|}{ 3. Recommended Procurement Strategy(coordinate with project CGI interface Engineer or BTR): NA } \\
\hline \multicolumn{4}{|c|}{ 等 } \\
\hline \multicolumn{4}{|c|}{$\begin{array}{l}\text { 1. Question \#1: Is the ltem subject to design or specification requirements that are unique to nuclear facilities or activities? } \\
\text { [ ] YES (the Item is not commercial grade) } \\
\text { [X] NO (continue) }\end{array}$} \\
\hline \multicolumn{4}{|c|}{$\begin{array}{l}\text { 2. Question \#2: Is the ltem used in applications other than nuclear facilities or activities? } \\
\text { [ ] NO (the item is not commercial grade) } \\
\text { [X] YES (continue) }\end{array}$} \\
\hline
\end{tabular}


Commercial Grade Item Upgrade Dedication Form

ECN No. NA CGINo. CGI-SNF-D-46-1-P4-015

Title: WORCESTER SOLENOW-ACTUATED GAS-OPERATED MCO ISOLATION VALVES
Rev. No. 0

Page 2 of 13

SNS. 3890

3. Question \#3: Is the Item ordered from manufacturer/supplier on the basis or specifications set forth in the manufacturers catalog?

[ ] NO (the ltem is not commercial grade)

[X] YES (continue)

[X] All three criteria have been satisfied. The ltem meets the definition of commercial grade.

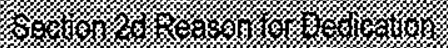

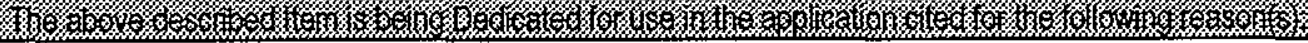

[X] Item is being purchased from a non ESL manufacturer supplier as commercial grade to be used in a Safety Class application.

[ ] Item is being purchased from a non ESL manufacturer supplier as commercial grade to be used in a Safety Significant application.

[ ] Item was purchased from a non ESL manufacturer supplier as commercial grade to be used in a Safety Class application.

[ ] Item was purchased from a non ESL manufacturer supplier as commercial grade to be used in a Safety Significant application.

[ $]$ Other ('like-for-like', similar, substitution, replacement evaluation)

2.

A. Part/Component Safety Function:

1. Maintain an intact pressure boundary/confinement.

2. Isolate the MCO in the event of loss of vacuum to reduce air inleakage or loss of He, prevent an $\mathrm{H}_{2}$ explosion.

3. Maintain critical function during and after seismic event.

B. Part/Component Functional Mode:

Safety Function \#1:

I I Active - Mechanical or Electrical change of state is required to occur for the component to perform its safety function

[X] Passive - Change of state is not required for the component to perform its safety function

Safety Function \#2:

[X] Active-Mechanical or Electrical change of state is required to occur for the component to perform its safety function.

[ ] Passive - Change of state is not required for the component to perform its safety function

Safety Function \#3:

[ ] Active - Mechanical or Electrical change of state is required to occur for the component to perform its safety function.

[X] Passive - Change of state is not required for the component to perform its safety function

C. Host Component Safety Function (if applicable):

1. NA

2.

3. 


\section{Error}

An error occurred while processing this page. See the system log for more details. 

ISOLATION VALVES

\begin{tabular}{|c|c|c|c|c|}
\hline $\begin{array}{l}\text { Critical Characteristics Verification } \\
\text { Document: Vendor Specifications; } \\
\text { HNF-SD-SNF-SEL-002, Rev. } 4 .\end{array}$ & Acceptance Criteria/Tolerances & $\begin{array}{l}\text { Acceptance } \\
\text { Method }\end{array}$ & ID & Function \\
\hline \multicolumn{5}{|c|}{ 1. Item Identification Critical Characteristics (necessary for reasonable assurance that the Item delivered is the ltem specified) } \\
\hline Manufacturer & Worcester Controls & 1, IN & $\mathbf{x}$ & \\
\hline Valve Part Number & 1"5966RTBW4 & $1, \mathbb{I N}$ & $\mathbf{x}$ & \\
\hline $\begin{array}{l}\text { Actuator Part Number (Includes } \\
\text { SOV) }\end{array}$ & 151939SWM2120PBC & $1, \mathbb{N}$ & $\mathbf{x}$ & \\
\hline Nameplate Data of Valve & Per Vendor Manual & 1, IN & $\mathbf{x}$ & \\
\hline $\begin{array}{l}\text { Nameplate Data of Actuator } \\
\text { (Includes SOV) }\end{array}$ & $\begin{array}{l}\text { Pèr Vendor Manual. To include "R6" } \\
\text { (upper right corner) }\end{array}$ & 1, IN & $\mathbf{x}$ & \\
\hline \multicolumn{5}{|c|}{ 2. Physical Critical Characteristics (for reasonable assurance that the Item delivered is the Item specified) } \\
\hline Valve Body Material & Stainless Steel & $1, T$ & $\mathbf{x}$ & \\
\hline Configuration/Mounting & $\begin{array}{l}\text { Integral Actuator/Nalve Assembly. } \\
\text { Transverse actuator mounting. Black } \\
\text { recessed override button. }\end{array}$ & $1, \mathrm{IN}$ & $\mathbf{x}$ & \\
\hline Valve Seat & Reinforced TFE cavity filler seat & $1, \mathrm{IN}$ & $\mathbf{x}$ & \\
\hline \multicolumn{5}{|c|}{ 3. Performance Critical Characteristics (for reasonable assurance that the Item will perform its intended safety function(s)) } \\
\hline GOV Pressure Boundary & $\begin{array}{l}\text { Pressure test at } 165 \text { psig (Zero leakage) } \\
\text { Note } 3\end{array}$ & $1, T$ & & $\mathbf{x}$ \\
\hline GoV Seat leakage & $\begin{array}{l}\text { Pressure test to } 150 \text { psig ( }<10^{-4} \mathrm{cc} \\
\mathrm{He} / \mathrm{sec} \text { Bubbletight Standards) }\end{array}$ & $1, \mathrm{~T}$ & & $\mathbf{x}$ \\
\hline GOV Fail safe position & $\begin{array}{l}\text { Valve fails closed on loss of air pressure } \\
\text { or electrical power to the solenoid. } \\
\text { Stroke time less than } 1 \text { second }\end{array}$ & $1, T$ & & $\mathbf{x}$ \\
\hline Insulation Resistance & Greater than 10 Megohm resistance & $1, \mathrm{~T}$ & & $\mathbf{x}$ \\
\hline Solenoid inrush current & Nominal 0.4 amp @ 120 VAC & $1, T$ & & $\mathbf{x}$ \\
\hline Solenoid holding current & Nominal 0.2 amp @ 120 VAC & $1, T$ & & $\mathbf{x}$ \\
\hline $\begin{array}{l}\text { Current carrying capability of } \\
\text { contacts }\end{array}$ & $\begin{array}{l}\text { Nominal } 5 \text { amps @ } 120 \text { VDC, Less than } \\
0.5 \text { V drop across contacts }\end{array}$ & $1, T$ & & $\mathbf{x}$ \\
\hline Environmental & Note 1 & & & \\
\hline Seismic Condition A & Note 2 & $1, T$ & & $\mathbf{x}$ \\
\hline
\end{tabular}


4. Notes and Legend:

1. These valves have coro-lube (nickel-acetate), acetal resin, and NEMA Enclosed Solenoids, these materials are not subject to degradation at $40^{\circ} \mathrm{F}$ and $60 \% \mathrm{RH}$ or $115^{\circ} \mathrm{F}$ and $22 \% \mathrm{RH}$ and are suitable for Condition B Application.

2. Maintain critical function during and after Seismic event. $W$ 441-P4, Rev. 2, Appendix L, page L-6, provides a seismic testing plan for these components at a (TBD) seismic spectra.

Acceptance Method:

1. Special Test and Inspection

1, IN for Inspection

1,T for Test

2. Commercial Grade Survey

3. Source Verification

4. Vendor/ltem History

3. Pressure test at $110 \%$ of design accident condition pressure of 150 psig.

Approvals:

Designated Engineer:

Design Authority.

QA Engineer.

DDtan 12/21198 


\begin{tabular}{|c|c|c|}
\hline \multicolumn{3}{|c|}{$\begin{array}{c}\text { WORKSHEET } 1 \\
\text { DETERMINATION OF FAILURE MECHANISMSMMODES }\end{array}$} \\
\hline \multicolumn{3}{|c|}{ 17. } \\
\hline \multicolumn{2}{|l|}{$\begin{array}{l}\text { Typical Failure } \\
\text { Mechanisms }\end{array}$} & $\begin{array}{l}\text { Applicable to Component } \\
\text { under Evaluation }\end{array}$ \\
\hline Fracture & $\begin{array}{l}\text { Separation of a solid accompanied by little or no } \\
\text { macroscopic plastic deformation. }\end{array}$ & $\begin{array}{l}\text { Yes [ ] No [X]; if Yes, indicate failure } \\
\text { Mode }\end{array}$ \\
\hline Corrosion & $\begin{array}{l}\text { The gradual deterioration of a material due to } \\
\text { chemical or electrochemical reactions, such as }\end{array}$ & $\begin{array}{l}\text { Yes [ ] No [X]; if Yes, indicate failure } \\
\text { Mode }\end{array}$ \\
\hline Erosion & $\begin{array}{l}\text { oxidation, between the material and its environment. } \\
\text { Destruction of materials by the abrasive action of } \\
\text { moving fluids, usually accelerated by the presence } \\
\text { of solid particles carried with the fluid. }\end{array}$ & $\begin{array}{l}\text { Yes [ ] No [X]; If Yes, indicate failure } \\
\text { Mode. }\end{array}$ \\
\hline Open Circuit & $\begin{array}{l}\text { An electrical circuit that is unintentionally broken so } \\
\text { that there is no complete path for current flow. }\end{array}$ & $\begin{array}{l}\text { Yes }[X] \text { No [ ]; if Yes, indicate failure } \\
\text { Mode Open or short in Motor actuator } \\
\text { circuit }\end{array}$ \\
\hline Short Circuit & $\begin{array}{l}\text { An abnormal connection by which an electrical } \\
\text { current is connected to ground, or to some }\end{array}$ & $\begin{array}{l}\text { Yes [X] No [ ]; If Yes, indicate failure } \\
\text { Mode }\end{array}$ \\
\hline Blockage & $\begin{array}{l}\text { conducting body, resulting in excessive current flow. } \\
\text { Clogging of a filtering medium resulting in the } \\
\text { inability to perform its purification function or } \\
\text { blockage of flow. }\end{array}$ & $\begin{array}{l}\text { Yes [ I No [X]; if Yes, indicate failure } \\
\text { Mode }\end{array}$ \\
\hline Seizure & $\begin{array}{l}\text { Binding of a normally moving item through excessive } \\
\text { pressure, temperature, friction, jamming. }\end{array}$ & $\begin{array}{l}\text { Yes [X] No [ ]; If Yes, indicate failure } \\
\text { Mode Structural failure or seizure of } \\
\text { valve/disc }\end{array}$ \\
\hline \multirow{2}{*}{$\begin{array}{l}\text { Unacceptable } \\
\text { Vibration }\end{array}$} & Mechanical oscillations produced are beyond the & Yes [ ] No [X]; If Yes, indicate failure \\
\hline & $\begin{array}{l}\text { defined permissible limits due to unbalancing, poor } \\
\text { support, or rotation at critical speeds. }\end{array}$ & Mode \\
\hline Loss of Properties & $\begin{array}{l}\text { A loss of mechanical and physical properties of a } \\
\text { material due to exposure to high temperatures, } \\
\text { radiation exposure. }\end{array}$ & $\begin{array}{l}\text { Yes [ ] No [X]; if Yes, indicate failure } \\
\text { Mode }\end{array}$ \\
\hline Excess Strain & $\begin{array}{l}\text { Under the action of excessive extemal forces the } \\
\text { material of the part has been deformed or distorted. }\end{array}$ & $\begin{array}{l}\text { Yes [ ] No [X]; If Yes, indicate failure } \\
\text { Mode. }\end{array}$ \\
\hline Mechanical Creep & $\begin{array}{l}\text { From prolonged exposure to high temperature and } \\
\text { stress, the object will show a slow change in its }\end{array}$ & $\begin{array}{l}\text { Yes [ ] No [X]; If Yes, indicate failure } \\
\text { Mode }\end{array}$ \\
\hline \multirow[t]{2}{*}{ Ductile Fracture } & $\begin{array}{l}\text { characteristics. } \\
\text { Fracture characterized by tearing of metal } \\
\text { accompanied by appreciable gross plastic } \\
\text { deformation. }\end{array}$ & $\begin{array}{l}\text { Yes [ ] No [X]; If Yes, indicate failure } \\
\text { Mode. }\end{array}$ \\
\hline & 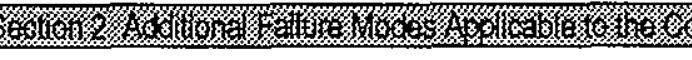 & 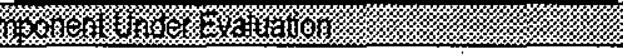 \\
\hline \multicolumn{3}{|c|}{ 1. $\quad$ Loss of air pressure. } \\
\hline \multicolumn{3}{|l|}{2.} \\
\hline \multicolumn{3}{|l|}{3.} \\
\hline \multicolumn{3}{|l|}{4.} \\
\hline \multicolumn{3}{|l|}{5.} \\
\hline
\end{tabular}




\section{CHECKLIST 1}

ACCEPTANCE METHOD 1

SPECIAL TESTIINSPECTION VERITICATION

\begin{tabular}{|c|c|c|c|c|}
\hline & & & & 3. \\
\hline $\begin{array}{l}\text { Item } \mathrm{D} \\
\text { Valve } \\
\text { Systen }\end{array}$ & $\begin{array}{l}\text { escript } \\
\text { s } \\
\text { \# } 4\end{array}$ & on: 1 "1 & MCO Solenoid Isolation & $\begin{array}{l}\text { Equip \#: GOV/SOV-1*03 \& 1*30 } \\
\text { Model \#: 1"5966RTBW4 with } \\
\text { 151939SWM2120PBC }\end{array}$ \\
\hline $\begin{array}{l}\text { Worc } \\
\text { P.O. } \\
33 \text { Lo } \\
\text { Marbe } \\
\text { (508) } \\
\text { P.O. \# }\end{array}$ & $\begin{array}{r}\text { Mar } \\
\text { ester } \\
\text { Box } 5 \\
\text { ck Dr. } \\
\text { orough } \\
481-4\end{array}$ & $\begin{array}{l}\text { ufactur } \\
8 \\
\text { ontrol } \\
\text { MA } \\
800\end{array}$ & $\begin{array}{l}\text { r (Address/Phone): } \\
1752\end{array}$ & Supplier (Address/Phone): \\
\hline & & $\begin{array}{l}\text { Post } \\
\text { Test }\end{array}$ & & \\
\hline$[\mathbf{X}]$ & 11 & [ ] & 1. Manufacturer & \\
\hline$[\mathbf{X}]$ & [ ] & [ ] & 2. Valve Part Number & \\
\hline$[\mathbf{X}]$ & [ ] & [ ] & 3. Actuator Part Numb & (Includes SOV) \\
\hline$[\mathbf{X}]$ & [ ] & [ ] & 4. Nameplate Data Of & alve \\
\hline$[\mathbf{X}]$ & [ ] & [ ] & 5. Nameplate Data of & tuator (Includes SOV) \\
\hline [ ] & {$[\mathbf{X}]$} & {$[$ ] $]$} & 6. Valve Body Material & \\
\hline$[\mathbf{X}]$ & [ ] & {$[$ ] } & 7. Configuration/Moun & \\
\hline$[\mathbf{X}]$ & I 1 & [ ] & 8. Valve Seat & \\
\hline [ ] & {$[\mathbf{X}]$} & {$[$ ] } & 9. Gov Pressure Bound & \\
\hline [ ] & {$[\mathbf{X}]$} & [ ] & 10. GoV Seat Leakage & \\
\hline [ ] & {$[\mathbf{X}]$} & [ ] & 11. GOV Fail Safe Posit & \\
\hline [ ] & {$[\mathbf{X}]$} & [ ] & 12. Insulation Resistanc & \\
\hline [ ] & {$[\mathbf{X}]$} & [ ] & 13. Solenoid Inrush Cur & \\
\hline [ ] & {$[\mathbf{X}]$} & [ ] & 14. Solenoid Holding $\mathrm{Cu}$ & ent \\
\hline [ ] & {$[\mathbf{X}]$} & [ ] & 15. Current Carrying Ca & ability of Contacts \\
\hline [ ] & {$[\mathbf{X}]$} & [ ] & 16. Seismic Condition A & \\
\hline
\end{tabular}



ISOLATION VALVES

\section{6.}

* See Attachment G of Desk Instruction for Sampling Size

Characteristic: Manufacturer

Sample Size*: All Items

Acceptance Criteria: Worcester Controls

Receipt Inspection Plan/ Report \#:

References (see Section 7):

Characteristic: Valve Part Number

Sample Size*: All Items

Acceptance Criteria: 1"5966RTBW4

Receipt Inspection Plan / Report \#:

References (see Section 7):

Characteristic: Actuator Part Number (Includes SOV)

Sample Size*: All Items

Acceptance Criteria: 151939SWM2120PBC

Receipt Inspection Plan / Report \#:

References (see Section 7):

Characteristic: Nameplate Data of Valve

Sample Size*: All Items

Acceptance Criteria: Per Vendor Manual

Receipt Inspection Plan / Report \#:

References (see Section 7): Manual PB 302-26, PB 451-22, PB 303-1

Characteristic: Nameplate Data of Actuator (Includes SOV)

Sample Size*: All Items

Acceptance Criteria: Per Vendor Manual. To include "R6" (upper right corner).

Receipt Inspection Plan / Report \#:

References (see Section 7): Manual PB 302-26, PB 451-22, PB 303-1

Characteristic: Configuration/Mounting

Sample Size*: All Items

Acceptance Criteria: Integral Actuator/Nalve Assembly. Transverse actuator mounting. Black recessed override button.

Receipt Inspection Plan / Report \#:

References (see Section 7): 
Characteristic: Valve Seat

Sample Size*: All Items

Acceptance Criteria: Reinforced TFE cavity filler seat

Receipt Inspection Plan / Report \#:

References (see Section 7):

\#

* See Attachment G of Desk Instruction for Sampling Size

Test To Be Performed by:

Number of Items to be Tested:

[ ] Purchaser

[ ] Supplier/Manufacturer**

Test/Inspection Location:

[ ] Other

Characteristic for Test: Valve Body Material

Acceptance Criteria: Stainless Steel

Sample Size*: Normal Sampling Size

Actual Test Value:

Test Plan and Report \#: References (see Section 7):

Characteristic for Test: GOV Pressure Boundary

Acceptance Criteria: Pressure Test at 165 Psig (Zero Leakage)

Sample Size*: Normal Sampling Size

Actual Test Value:

Test PIan and Report \#:

References (see Section 7):

Characteristic for Test: GOV Seat leakage

Acceptance Criteria: Pressure Test to 150 Psig $\left(<10^{-4} \mathrm{cc} \mathrm{He} / \mathrm{sec}\right.$ Bubbletight Standards)

Sample Size*: Normal Sampling Size

Actual Test Value:

Test Plan and Report \#: References (see Section 7):

\section{Characteristic for Test: GOV Fail Safe Position}

Acceptance Criteria: Valve fails closed on loss of air pressure or electrical power to the solenoid. Stroke time less than 1 second.

Sample Size*: Normal Sampling Size

Actual Test Value:

Test Plan and Report \#: References (see Section 7): 

ISOLATION VALVES

Characteristic for Test: Insulation Resistance

Acceptance Criteria: Greater Than 10 Megohm Resistance

Sample Size*: Normal Sampling Size

Actual Test Value:

Test Plan and Report \#: References (see Section 7):

Characteristic for Test: Solenoid Inrush Current

Acceptance Criteria: Nominal 0.4 amp @ 120 VAC

Sample Size*: Normal Sampling Size

Actual Test Value:

Test Plan and Report \#:

References (see Section 7):

\section{Characteristic for Test: Solenoid Holding Current}

Acceptance Criteria: Nominal 0.2 amp @ 120 VAC

Sample Size*: Normal Sampling Size

Actual Test Value:

Test Plan and Report \#: References (see Section 7):

\section{Characteristic for Test: Current Carrying Capability of Contacts}

Acceptance Criteria: Nominal 5 amps @ 120 VDC, less than $0.5 \mathrm{~V}$ drop across contacts.

Sample Size*: Normal Sampling Size

Actual Test Value:

Test Plan and Report \#: References (see Section 7):

Characteristic for Test: Seismic Condition A

Acceptance Criteria: Maintain critical function during and after seismic event.

Sample Size*: Normal Sampling Size

Actual Test Value:

Test Plan and Report\#:

References (see Section 7):

**If Supplier/Manufacturer or Other, Refer to CGI Checklist-2 for Support Information 
Commercial Grade Item Upgrade Dedication Form

ECN No. NA CGI No. CGI-SNF-D-46-1-P4-015

Title: WORCESTER SOLENOM-ACTUATED GAS-OPERATED MCO ISOLATION VALVES

\begin{tabular}{|l} 
Rev. No. 0 \\
Page 11 of 13 \\
SNI -3890
\end{tabular}

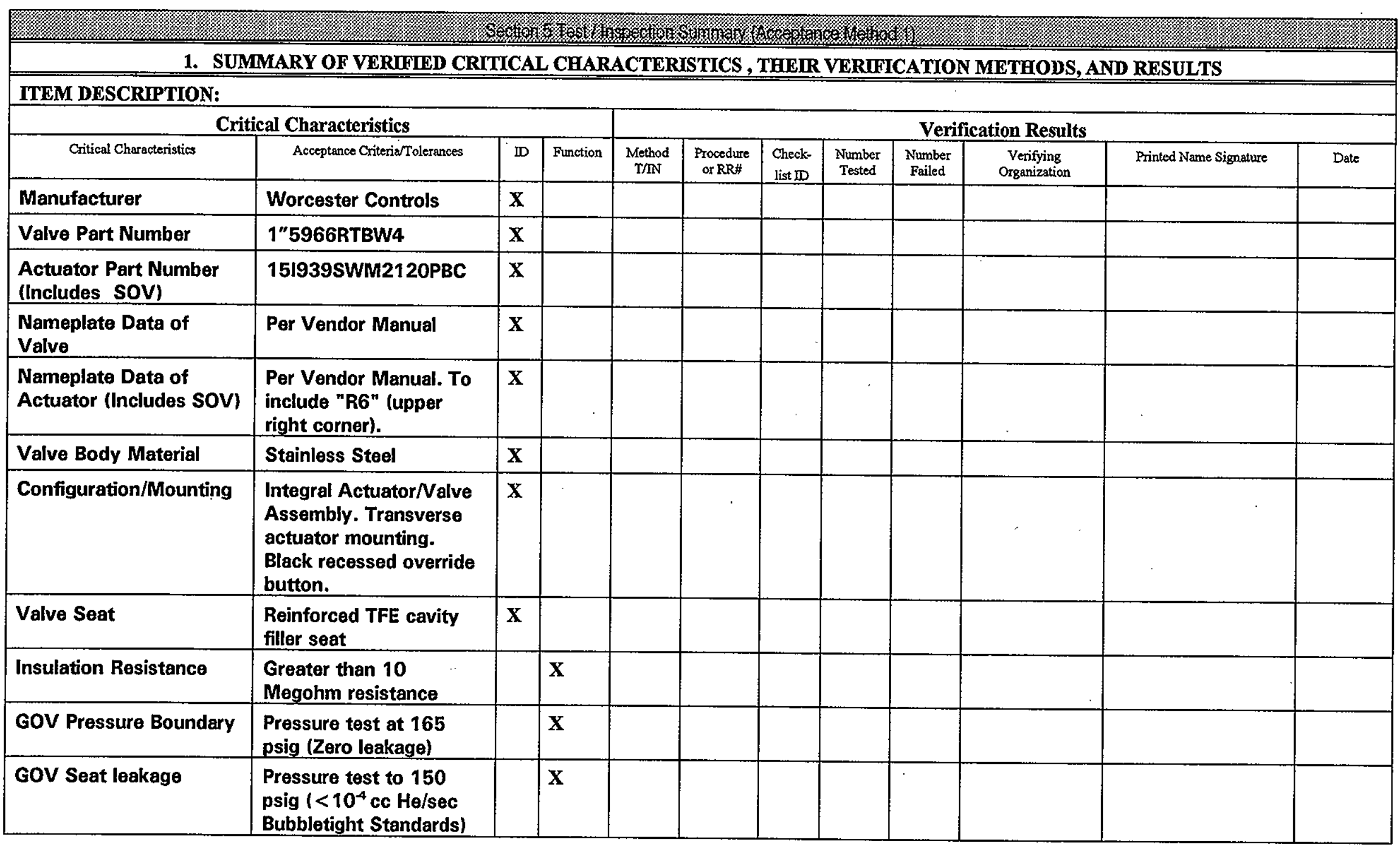


Commercial Grade Item Upgrade Dedication Form

ECN No. NA CGINo. CGI-SNF-D-46-1-P4-015

Title: WORCESTER SOLENOD-ACTUATED GAS-OPERATED MCO ISOLATION VALVES

\begin{tabular}{|l|}
\hline Rev. No. 0 \\
\hline Rage 12 of 13 \\
SNS. 3890
\end{tabular}

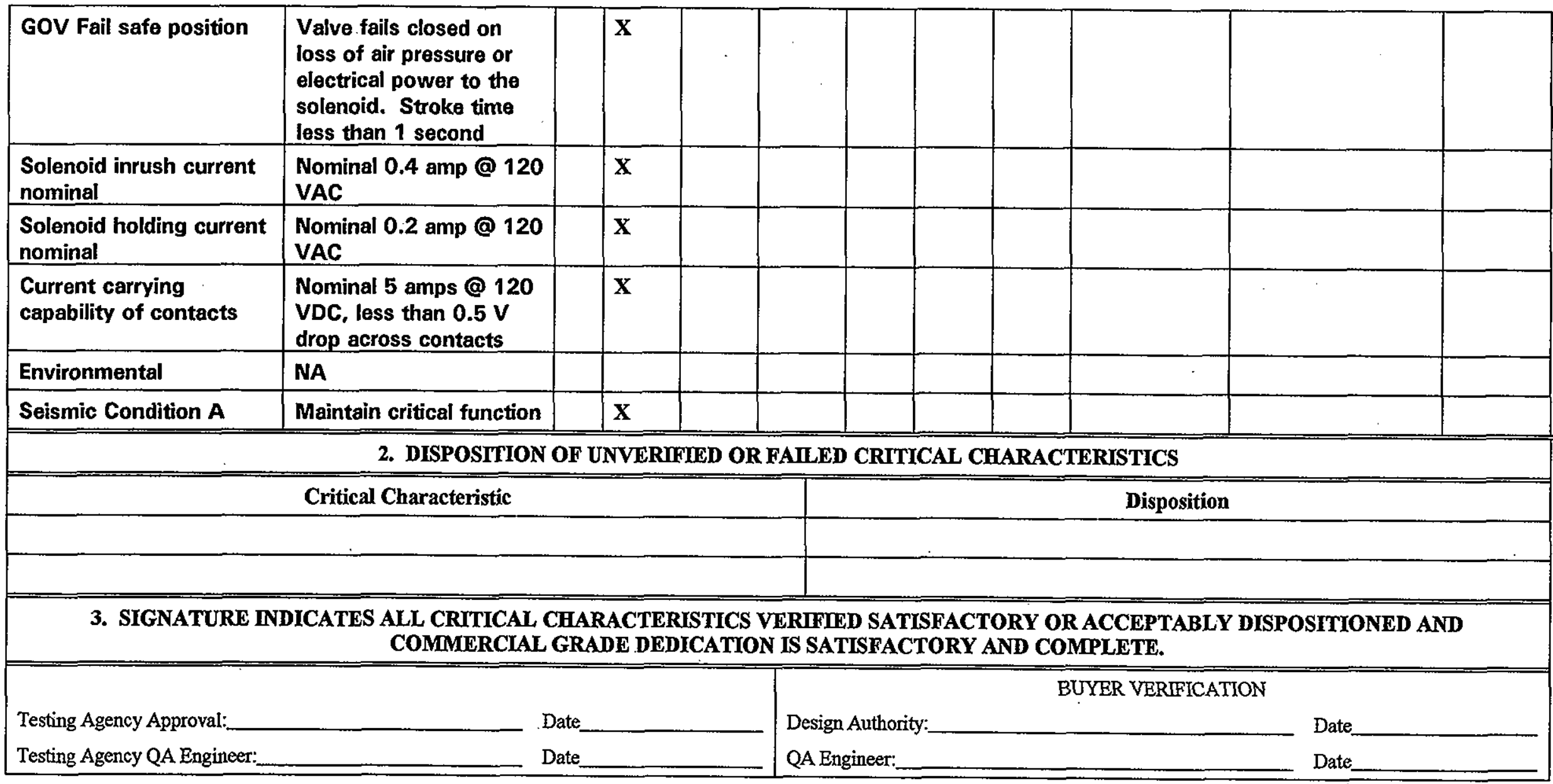


Commercial Grade Item Upgrade Dedication Form

ECN No. NA CGINo. CGI-SNF-D-46-1-P4-015

Title: WORCESTER SOLENOM-ACTUATED GAS-OPERATED MCO ISOLATION VALVES
Rev. No. 0

Page 13 of 13

SNF 3890

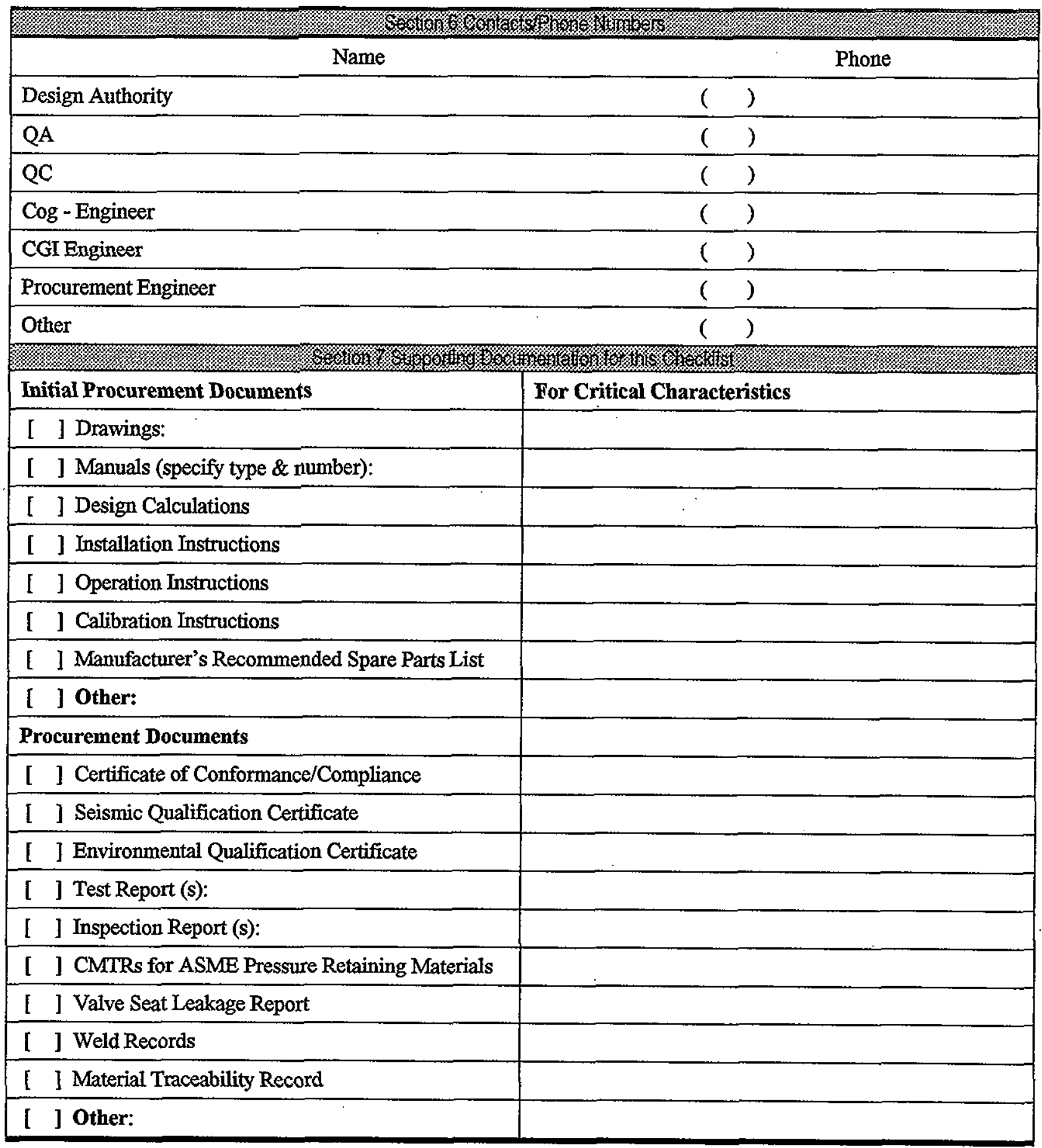

